\title{
Prototype of an Interface for Hyphenating Distillation with Gas Chromatography and Mass Spectrometry
}

\author{
Ya-Ru Tang, Hui-Hsien Yang, and Pawel L. Urban* \\ Department of Applied Chemistry, National Chiao Tung University, 1001 University Rd, Hsinchu 300, Taiwan
}

\begin{abstract}
Chemical analysis of complex matrices-containing hundreds of compounds-is challenging. Two-dimensional separation techniques provide an efficient way to reduce complexity of mixtures analyzed by mass spectrometry (MS). For example, gasoline is a mixture of numerous compounds, which can be fractionated by distillation techniques. However, coupling conventional distillation with other separations as well as MS is not straightforward. We have established an automatic system for online coupling of simple microscale distillation with gas chromatography (GC) and electron ionization MS. The developed system incorporates an interface between the distillation condenser and the injector of a fused silica capillary GC column. Development of this multidimensional separation (distillation-GC-MS) was preceded by a series of preliminary off-line experiments. In the developed technique, the components with different boiling points are fractionated and instantly analyzed by GC-MS. The obtained data sets illustrate dynamics of the distillation process. An important advantage of the distillation-GC-MS technique is that raw samples can directly be analyzed without removal of the non-volatile matrix residues that could contaminate the GC injection port and the column. Distilling the samples immediately before the injection to the GC column may reduce possible matrix effects-especially in the early phase of separation, when molecules with different volatilities co-migrate. It can also reduce losses of highly volatile components (during fraction collection and transfer). The two separation steps are partly orthogonal, what can slightly increase selectivity of the entire analysis.
\end{abstract}

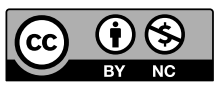

Copyright (C 2017 Ya-Ru Tang, Hui-Hsien Yang, and Pawel L. Urban. This is an open access article distributed under the terms of Creative Commons Attribution License, which permits use, distribution, and reproduction in any medium, provided the original work is properly cited and is not used for commercial purposes.

Please cite this article as: Mass Spectrom (Tokyo) 2017; 6(2): S0061

Keywords: distillation, gas chromatography, gasoline, hyphenated techniques, online sample preparation

(Received August 12, 2016; Accepted January 11, 2017)

\section{INTRODUCTION}

The term "petroleum" is used to describe both unprocessed crude oil as well as crude oil refining products. Petroleum is a complex mixture of hydrocarbons with different carbon numbers. It also contains small amounts of nitrogen, oxygen, sulfur, and metal compounds. ${ }^{1)}$ Density, viscosity, and boiling points are the main physical properties determined for petroleum and its components. Depending on the percentage of the low-boiling-point $\left(<200^{\circ} \mathrm{C}\right)$ or lowspecific-gravity components, petroleum can be classified as light or heavy. Light petroleum contains larger share of low-boiling-point components (typically, 75\%) than heavy petroleum (typically, 25\%). ${ }^{1)}$ The fractions obtained while refining light petroleum are used to synthesize numerous consumer materials such as gasoline, asphalt and plastics. In fact, in the middle of the 19th century, gasoline was an unwanted by-product of petroleum distillation. ${ }^{2,3)}$ Gasoline is a mixture of more than 100 compounds, mainly hydrocarbons with 4-12 carbon atoms. Their boiling points range from 35 to $200^{\circ} \mathrm{C}^{1,4-6)}$
Distillation is commonly used for separation of mixtures of volatile compounds due to differences in their boiling points. It is used on an industrial scale for fractionation of petroleum (in refineries) as well as in the laboratory-scale sample preparation (e.g., before chemical analysis of complex samples). Beside simple distillation, several customized variants of this technique (including fractional distillation, ${ }^{7)}$ vacuum distillation, ${ }^{8,9)}$ molecular distillation, ${ }^{10)}$ and simulated distillation ${ }^{11,12)}$ ) are in common use. For example, fractional distillation takes advantage of several vapor condensation steps taking place in one apparatus, providing enhanced separation of volatile components because of the high number of theoretical plates. ${ }^{7}$

Chemical analysis of complex matrices-containing tens or hundreds of compounds-is challenging. Chromatographic separations, frequently conducted prior to mass spectrometry (MS) detection, simplify compound identification and quantification-especially if low-resolution mass spectrometers are used. However, in some cases, a one-step separation before MS is not sufficient. Two-dimensional separation techniques provide an efficient means to reduce complexity of samples introduced to the MS systems. Such

*Correspondence to: Pawel L. Urban, Department of Applied Chemistry, National Chiao Tung University, 1001 University Rd, Hsinchu 300, Taiwan, e-mail: plurban@ nctu.edu.tw 


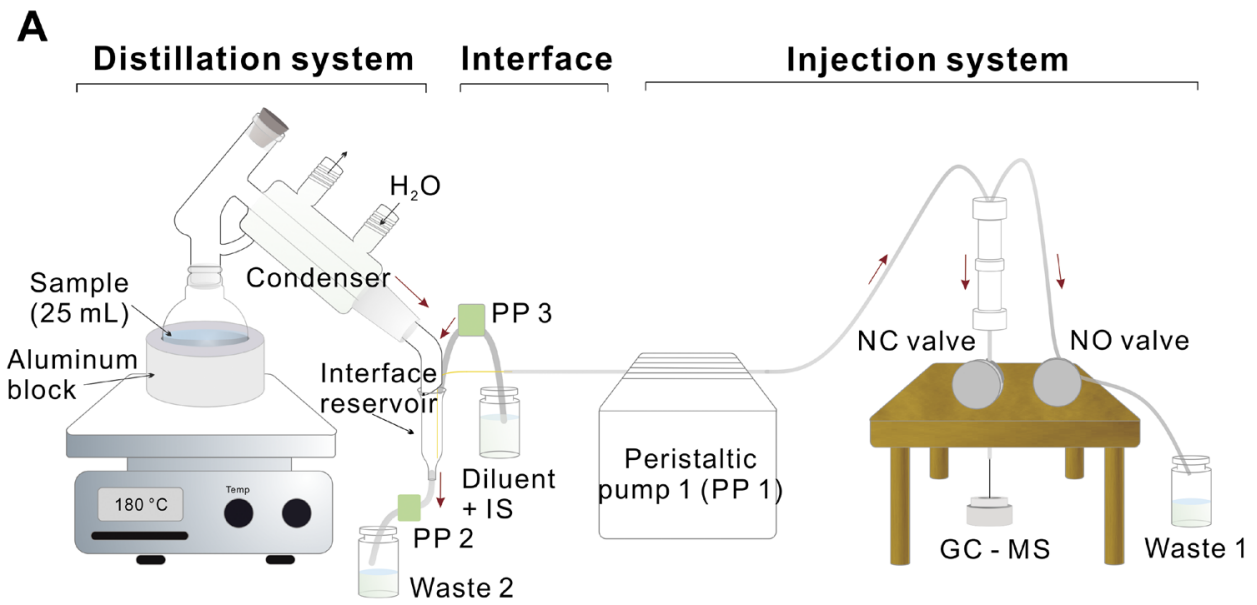

B

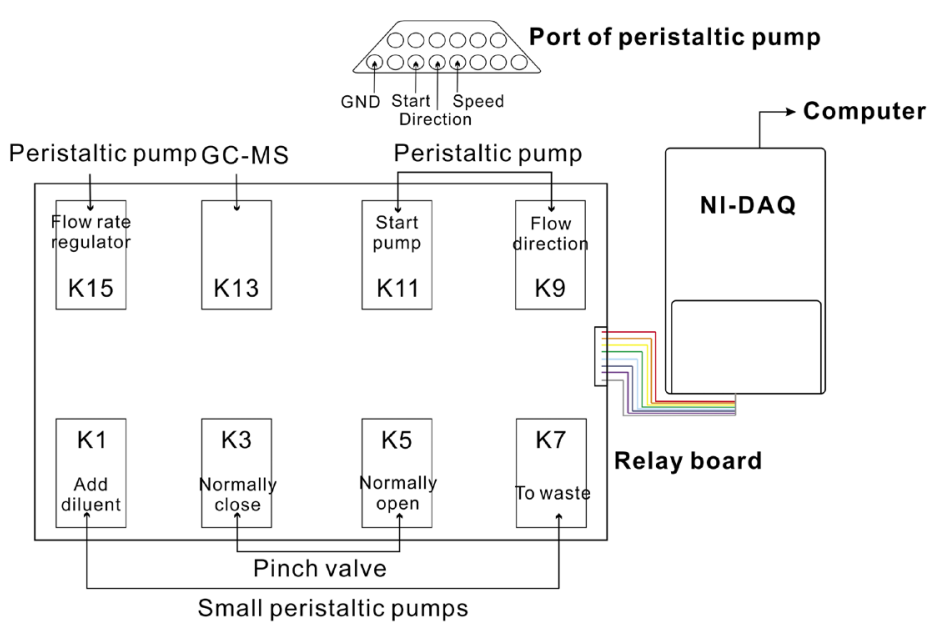

Fig. 1. Schematic illustration of the multi-dimensional online separation system incorporating simple distillation and gas chromatography: (A) distillation system interfaced with gas chromatograph; (B) simplified schematic of the electronic control unit.

comprehensive separations are particularly useful when analyzing petroleum and its derivative products. ${ }^{13-15)}$ Complex mixtures of volatile compounds-including gasolinecan be fractionated by distillation techniques. Analysis of distillate fractions by MS could potentially yield results with lower complexity than analysis of unprocessed gasoline samples. However, despite some developments (e.g., distillate monitoring by reversed-phase membrane inlet mass spectrometry ${ }^{16)}$ ), online coupling of conventional distillation apparatuses with standard analytical instruments (chromatographs, mass spectrometers) has so far been problematic.

Here we propose an automated interface enabling hyphenation of simple microscale distillation with gas chromatography (GC) coupled with electron ionization MS. The proposed interface enables seamless transfer of gasoline distillate fractions into the injection port of the gas chromatograph. Such hyphenation adds one more dimension to the conventional GC-MS analysis workflows, facilitating analyses of complex samples in single runs, without any pretreatment. It reduces the risk of contamination of the chromatographic system (injection port, column) by non-volatile contaminants present in complex samples.

\section{EXPERIMENTAL}

\section{Materials}

Gasoline (research octane number: 92) was purchased from a local gas station (Hsinchu, Taiwan). Hexane (used in the initial tests) was purchased from Merck (Darmstadt, Germany). Ethanol (diluent), D-limonene and $\gamma$-terpinene were purchased from Sigma-Aldrich (St. Louis, MO, USA).

\section{Instrumentation}

The proposed system for online coupling of simple microscale distillation with GC-MS is illustrated in Fig. 1. The system incorporates an interface reservoir set between the distillation condenser (cat. No. D151814; Synthware Glass, Pleasant Prairie, WI, USA) and the injector of a fused-silica capillary GC column (length: $60 \mathrm{~m}$ in the off-line tests, or $30 \mathrm{~m}$ in the online tests; ID: $0.53 \mathrm{~mm}$; stationary phase: $5 \%$ polydiphenyl/95\% polydimethyl siloxane; cat. No. 25389 or 25305-U; Supelco, PA, USA). The funnel-shaped interface reservoir (height: $44 \mathrm{~mm}$; inner diameter: $10 \mathrm{~mm}$, diameter of the collar (upper part): $12 \mathrm{~mm}$; diameter of the outlet section (lower part): $1 \mathrm{~mm}$ ) was fabricated by the glass blowing workshop in the National Tsing Hua University (Hsinchu, Taiwan). Very small volumes of sample condensate are automatically transferred from the interface into an intermediate tubing (ID: $0.25 \mathrm{~mm}$; OD: $2.07 \mathrm{~mm}$; cat. No. SC0002; IDEX Health \& Science, WA, USA) by a peristaltic pump 
(Ismatec ISM936D, IPC Series, 8 channels; IDEX Health \& Science). Two pinch valves (12 V DC, P/N 075P2NO12-01S and P/N 075P2NC12-01S; Bio-Chem Fluidics; Booton, NJ, USA) direct several microliters of the condensate to the GC injector. This part of the system has been adapted from the previous system used for monitoring reactions and extractions. ${ }^{17,18)}$ The distillate fraction components were separated by GC (TRACE GC; Thermo Fisher Scientific, Waltham, MA, USA), and detected by an electron ionization quadrupole mass spectrometer (ISQ; Thermo Fisher Scientific). Ions in the $\mathrm{m} / \mathrm{z}$ range $25-200$ were monitored over $3.5 \mathrm{~min}$ (or $3.9 \mathrm{~min}$ in the off-line test and first online test). The initial GC temperature was $50^{\circ} \mathrm{C}$ (or $35^{\circ} \mathrm{C}$ in the off-line test and first online test). It was ramped up to $170^{\circ} \mathrm{C}$ (or $180^{\circ} \mathrm{C}$ in the off-line test and first online test) after $1 \mathrm{~min}$ of separation at a rate of $40^{\circ} \mathrm{C} \mathrm{min}^{-1}$. In order to minimize possible column and detector overloading during the GC-MS analyses, the split ratio was set to 100 (or 200 in the off-line test and first online test), while the carrier gas (helium) flow rate was set to $1 \mathrm{~mL} \mathrm{~min}^{-1}$ (or $2.5 \mathrm{~mL} \mathrm{~min}^{-1}$ in the off-line test and first online test). Moreover, a plug of glass wool was placed in the liner of the GC injection port to accommodate injection of large volume samples $(\sim 2 \mu \mathrm{L})$. The transfer of the distillate fraction aliquots from the condenser to the GC injector was controlled by a script created in the LabView software (version 2015; National Instruments, Austin, TX, USA), a DAQ NI USB-6343 electronic interface (cat. No. 781439-01; National Instruments), and a relay board (R16; Shenzhen Keyes DIY Robot, Shenzhen, China).

\section{Data acquisition and analysis}

The GC-MS data were acquired using the Xcalibur software (Thermo Fisher Scientific). The total ion currents (TICs) and the extracted ion currents (EICs) were exported from Thermo Xcalibur 2.1 Qual Browser (Thermo Fisher Scientific) to ASCII files. The time resolution was then set to $0.01 \mathrm{~min}$ to enable further processing. The chromatography data sets were then arranged into matrices for display in two-dimensional plots using OriginPro software (version 8; OriginLab, Northampton, MA, USA).

\section{RESULTS AND DISCUSSION}

\section{Off-line tests of distillation-GC-MS coupling}

The development of the multidimensional online separation (distillation-GC-MS) was preceded by a series of preliminary off-line experiments. The gasoline sample $(\sim 25 \mathrm{~mL})$ was loaded into a $50-\mathrm{mL}$ flask. In the off-line test, the flask was heated to $110^{\circ} \mathrm{C}$. The hot plate temperature was gradually increased up to $180^{\circ} \mathrm{C}$ (with a $20^{\circ} \mathrm{C}$ increment). Using this temperature program, components with different boiling points were fractionated. The fractions obtained manually $(1-2 \mathrm{~mL})$ were then analyzed by GC-MS without prior dilution (injection volume: $\sim 2 \mu \mathrm{L}$ ). The collected data sets highlight the dynamics of the distillation process (Fig. 2). The successive MS chromatograms reveal groups of numerous analyte peaks. The average retention times of the groups of peaks increase over time-from 1.0-2.5 min (fraction 1) to $2.5-4.5 \mathrm{~min}$ (fraction 8 ). As expected, the components with low boiling points (around 1-2 min) condensed faster than the components with high boiling points ( $4 \mathrm{~min})$. Overall, the TICs reveal the presence of $30-40$ features.

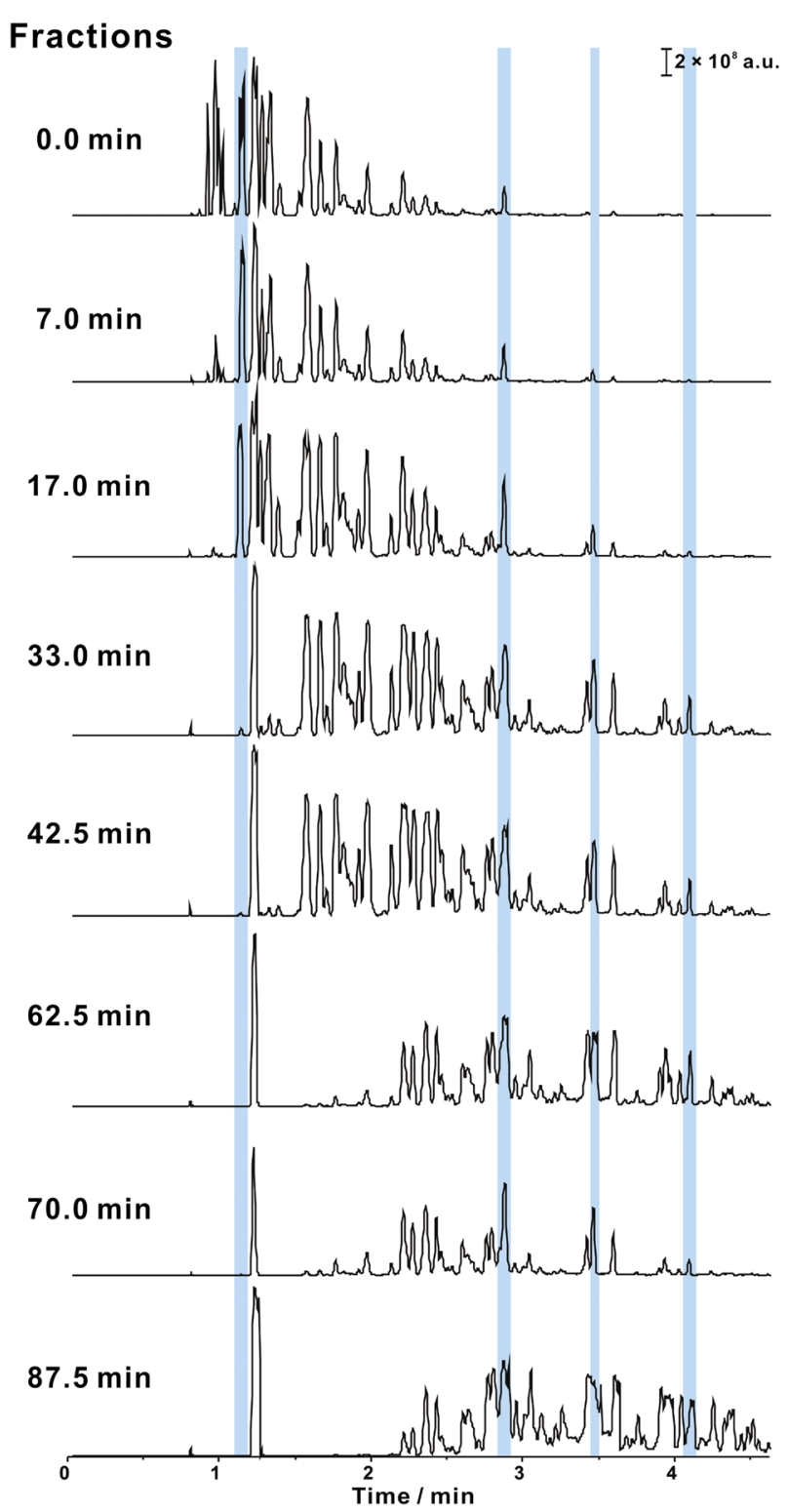

Fig. 2. Off-line GC-MS analysis of fractions of gasoline obtained during simple distillation. The blue bars highlight four chromatographic (TIC) features that clearly change in the course of distillation.

The TICs and EICs of the signals of interest were converted to $2 \mathrm{D}$ maps relating ion signal intensities with distillation times and retention times (Fig. 3). These 2D maps confirm usefulness of coupling distillation with GC-MS. When viewing the $2 \mathrm{D}$ maps, one can realize that if the dark spots were arranged only along the diagonal lines, it would mean that the components could be separated by either distillation or GC-MS, and that there would be no benefits from the distillation-GC coupling. However, several signals (dark spots in Fig. 3) are located outside the diagonal line. Interestingly, beside the early elution of the molecules with low boiling points from the condenser, they also produce more low $\mathrm{m} / \mathrm{z}$ fragments than the molecules with high boiling points (Fig. 3).

\section{Demonstration of online distillation-GC-MS}

Following the off-line proof-of-concept experiment (Fig. 2 ), we further attempted coupling the distillation step online 


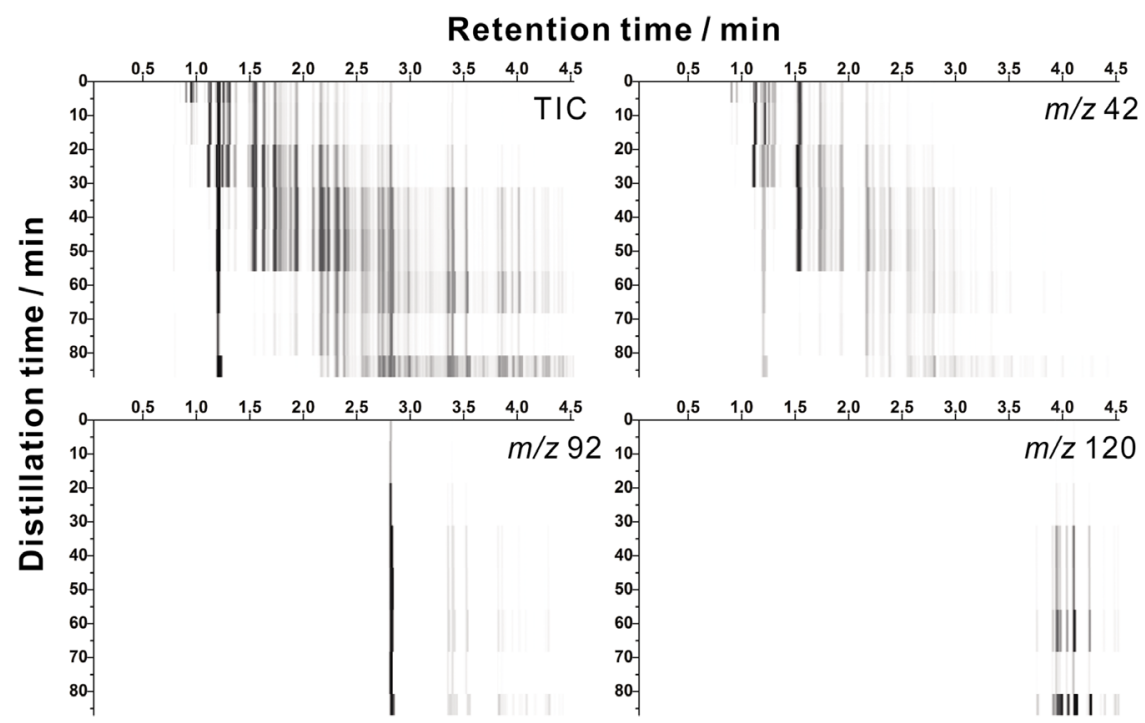

Fig. 3. Two-dimensional plots relating signal intensity with condensation time and GC-MS retention time: TIC and EICs for $m / z 42,92$, and 120 (selected ions related to gasoline hydrocarbons). This figure presents the same data set as Fig. 2.

with GC-MS analysis (Fig. 1). Two major impediments of coupling distillation with GC-MS are: (i) non-constant flow of the condensate, and (ii) requirement to match the duration of the GC separation with the time scale of the distillation process. They are addressed by incorporating an additional microfluidic pump in the interface chamber (between condenser and GC inlet) to remove excess of condensate, and by optimizing the GC separation method to shorten the analysis, and to enable collection of multiple data points during the whole distillation process ( $70 \mathrm{~min})$.

Overall, to achieve online distillation-GC-MS coupling, we incorporated two additional microfluidic pumps (PP2 and PP3; RP-Q1; Takasago Fluidic Systems, Nagoya, Japan) in the interface chamber (between condenser and GC inlet) to introduce a diluent and standard compounds, and to remove excess of condensate. PP3 optionally delivers the diluent (ethanol solution of $10^{-5} \mathrm{M}$ limonene and $10^{-5} \mathrm{M}$ terpinene) at a rate of $145 \mu \mathrm{L} \mathrm{min}^{-1}$, while PP2 removes the excessive amounts mixture of the diluent and distillate at a rate of $131 \mu \mathrm{L} \mathrm{min}^{-1}$. In order to fit different components into the interface, holders were manufactured of ABS polymer by 3D printer (UP Plus 2; Beijing TierTime Technology, Beijing, China). These holders fixed the positions of the pumps and interface reservoir.

Similarly to the off-line experiment, the result of the first online distillation-GC-MS analysis also reveals the dynamics of the distillation process (Fig. 4). The four TIC chromatograms represent GC-MS analysis of the fractions leaving the condenser at 33.0, 42.5, 62.5, and $70.0 \mathrm{~min}$. First online analysis of earlier and later fractions is problematic because the distillate flow rate at the condenser outlet is not constant. In the above first online experiment, the diluent solution was not dispensed to the interface reservoir by PP3 (cf. Fig. 1). The experimental parameters (e.g., temperature program, column length, split flow ratio, temperature setting on the hot plate) were same as those in the off-line test.

The second online test (with diluent and hot plate temperature program) was conducted following further optimization of the system (Fig. 5). Importantly, to shorten the analysis time, the column length was reduced from 60 to $30 \mathrm{~m}$. The initial and final temperatures of the GC program

\section{Fractions}

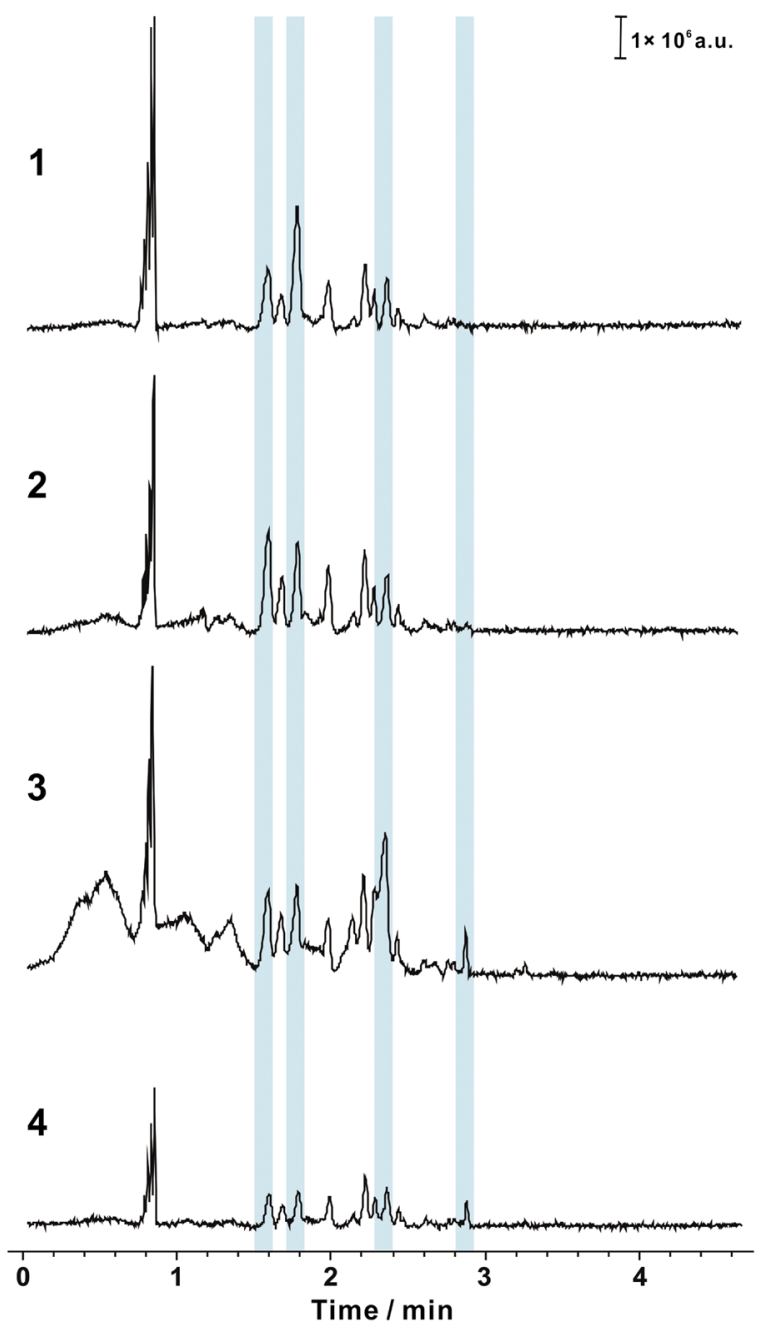

Fig. 4. Gas chromatograms (TIC) obtained during online coupling of microscale distillation with GC-MS, applied to analysis of a gasoline sample $(25 \mathrm{~mL})$ - first test without fraction dilution and without automated temperature control. The blue bars highlight four chromatographic features that clearly change in the course of distillation. 


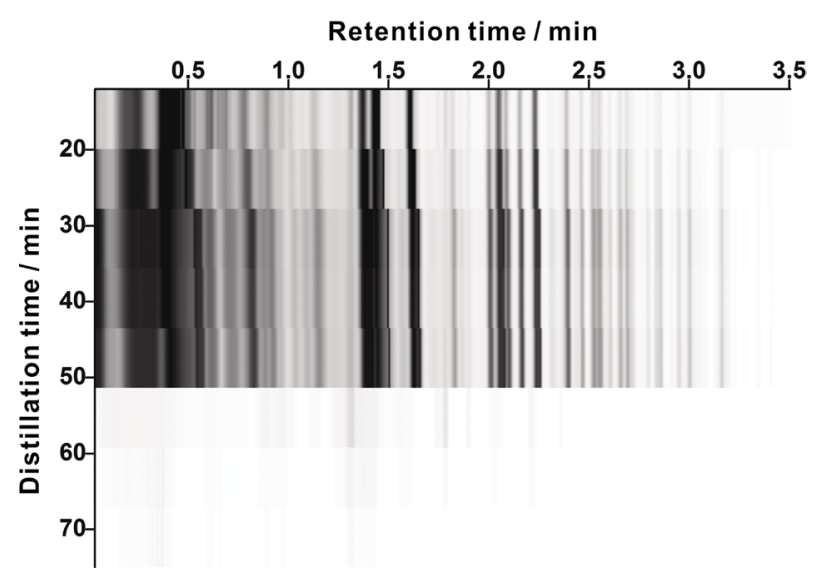

Fig. 5. Two-dimensional plot (TIC) obtained during online coupling of microscale distillation with GC-MS, applied to analysis of a gasoline sample $(25 \mathrm{~mL})$ - second test with fraction dilution and with automated temperature control.

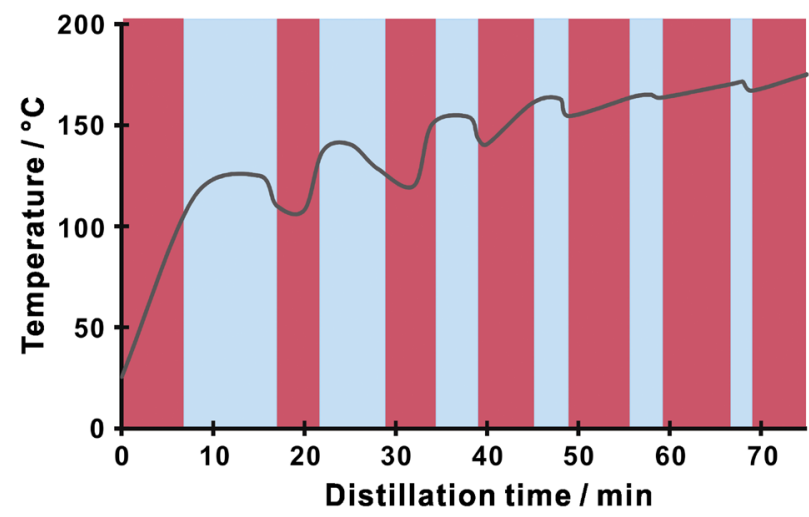

Fig. 6. Temperature program applied by the hot plate during distillation (second online test). Red zones: hot plate on. Blue zones: hot plate off.

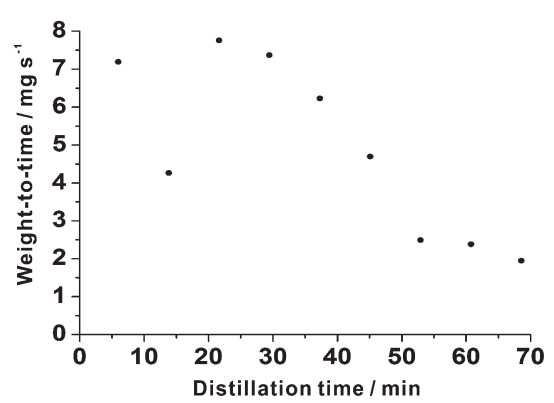

Fig. 7. Variability of the distillate output. Fractions were collected during 1-min intervals, and weighed. Experimental conditions are the same as in the second online test.

were changed from 35 and 180 to 50 and $170^{\circ} \mathrm{C}$, respectively. To enable control of distillation temperature, the hot plate was set to $180^{\circ} \mathrm{C}$, and controlled by a power-switch tail (PN 80136; PowerSwitchTail.com). Temperature was raised by triggering this power-switch tail with $5 \mathrm{~V}$ pulses. This way, a temperature ramp could be applied to the sample subjected to distillation (Fig. 6). Moreover, in the second online analysis, a diluent was added to the condensate accumulating in the interface reservoir ( $c f$. Fig. 1).

The non-constant flow of condensate (Fig. 7) affects coupling of distillation with GC system. Addition of the diluent to the condensate fraction can partly mitigate this problem.

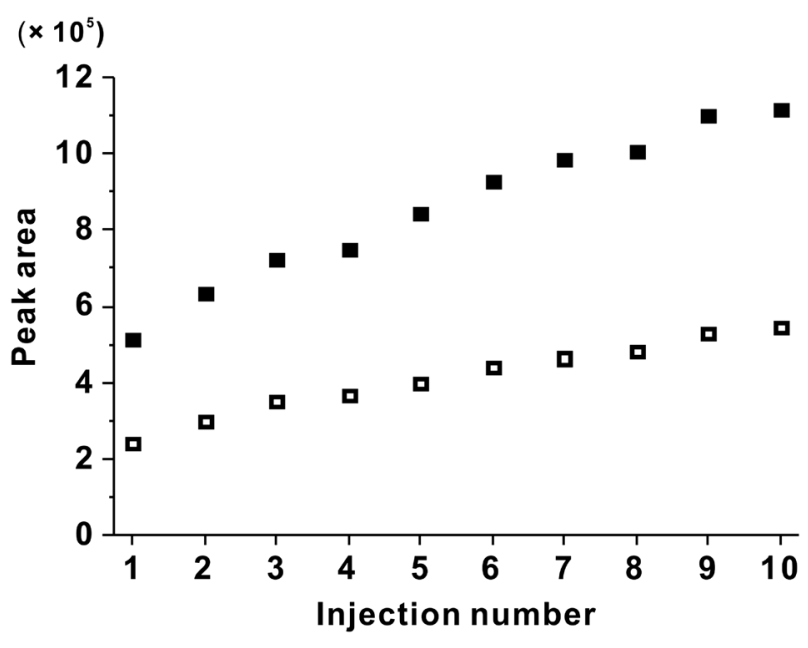

Fig. 8. Stability of the automated injection system (cf. Fig. 1). Peak areas of limonene and terpinene (standard spiked into diluent, $10^{-5} \mathrm{M}$ ). Labels: ( $\left.\mathbf{\square}\right)$ limonene; $(\boldsymbol{\square})$ terpinene.

However, it also decreases the signal intensities in ion currents, and deteriorates separation of compounds leaving the distillation apparatus (Fig. 5). Therefore, if the dilution system is used (as in the second online test), one cannot really take advantage of the separation capabilities of simple distillation. However, the remaining advantage is separation of volatile compounds from non-volatile sample components prior to GC-MS analysis. Please also note that, in the second online test, no signal was recorded after about $50 \mathrm{~min}$ of distillation. This is because, using the applied temperature program (Fig. 6), almost all volatile species have left the sample flask by that time. In this stage, even the addition of the diluent to the interface reservoir could not compensate for the small influx of the condensate.

The standard compounds, mixed with the diluent, were used to verify stability of the automated injection system before the online tests (Fig. 8). The system is generally stable but there is a drift of injection volume. This drift may be attributed to heating of the sample transfer tubing in front of the GC injection port. The increased temperature affects sample viscosity and the injected volume. If semiquantitative results are required, it may be possible to use the standard peaks to correct for the injection volume drift. However, in this early demonstration, the standard compounds were chemically unrelated to the analytes. For further application work, it is suggested to use isotopologue standards, which are chemically identical with the target analytes.

\section{Final remarks}

For years now, high-resolution and tandem mass spectrometry have been the "gold standard" in the characterization of complex samples such as petroleum and its products (e.g., refs. 19-21). However, high-resolution mass spectrometers are still inaccessible for many analytical laboratories. On the other hand, automation of sample preparation in analysis of complex hydrocarbon-rich matrices has received relatively little attention in the scientific literature. Distillation of petroleum products can be conducted by one of the accepted procedures. ${ }^{22)}$ The distilled fractions obtained in such procedures are occasionally analyzed by GC-MS to determine their composition. ${ }^{23)}$ While distillation can be 
done off-line, fraction collection and their manual transfer to gas chromatograph increase the risk of analyte losses (in the case of highly volatile compounds). In fact, since the introduction of the "purge-closed loop" and the related techniques, ${ }^{24)}$ online sample introduction to GC columns has been recognized as an efficient way of conducting analyses of complex and matrix-rich samples. This trend is reflected in the present work.

Although the feasibility of hyphenating distillation equipment with gas chromatograph has been demonstrated, the efficiency of fraction transfer should further be improved in future work. In one imaginable modification, the dilution factor could be sustained by real-time monitoring of the condensate flow-rate and dynamic adjustment of the diluent flow rate. Such routines require feedback from a sensor and additional programming. Moreover, the simple distillation implemented in this work does not warrant efficient separation of the sample components. Thus, a new version of the distillation-GC-MS system should implement fractionating distillation rather than simple distillation. In this prototype, we used a commercial research-grade electronic control system to co-ordinate distillation, dilution, sample injection, and analysis. To decrease the size and lower the cost of the distillation-GC-MS interface in the routine "screening" applications, it is appealing to use open-source electronic modules, which have recently been incorporated into the prototypes of various analytical instruments. ${ }^{25,26)}$ On the other hand, in the case of discovery-oriented applications, it would be desirable to couple this interface with detectors possessing a higher discriminatory power-i.e., high-resolution and tandem mass spectrometers-to enable identification and quantification of individual components.

\section{CONCLUSION}

Microscale distillation has been coupled online with GCMS to enable chemical profiling of complex samples without laborious sample preparation. An important advantage of this multidimensional analysis (distillation-GC-MS) over conventional one-step capillary GC (e.g., simulated distillation GC) is that raw samples can be directly analyzed without removal of the non-volatile matrix residues that could contaminate the GC-MS system. Distilling the samples right before the injection to the GC column reduces possible matrix effects (especially in the early phase of separation, when the separated components co-migrate in the GC column). Samples of gasoline containing non-volatile contaminants can be screened, possibly reducing the risk of contaminating the GC system. Moreover, the two separation steps are partly orthogonal, what-following further development-may increase the selectivity of the entire analysis process.

\section{Acknowledgements}

We would like to thank the National Chiao Tung University and the Ministry of Science and Technology of Taiwan (Grant No. MOST 104-2628-M-009-003-MY4) for the financial support of this work.

\section{REFERENCES}

1) J. G. Speight. Handbook of Petroleum Product Analysis. Wiley,
Hoboken, 2002.

2) D. Yergin. The Prize: The Epic Quest for Oil, Money \& Power. Free Press, New York, 2008.

3) A. M. Hochhauser. Gasoline and other motor fuels. Kirk-Othmer Encyclopedia of Chemical Technology 12: 386-435, 2014.

4) A. Y. El-Naggar, M. M. Al Majthoub. Study the toxic effects of aromatic compounds in gasoline in Saudi Arabia petrol stations. Int. J. Chem. Sci. 11: 106-120, 2013.

5) J.-Z. Zhang, J. Jin. The influence of common plastics on the identification of gasoline studied by GC-MS. Procedia Eng. 71: 372-376, 2014.

6) D. Swick, A. Jaques, J. C. Walker, H. Estreicher. Gasoline toxicity: Overview of regulatory and product stewardship programs. Regul. Toxicol. Pharmacol. 70(Suppl.): S3-S12, 2014.

7) D. W. Mayo, R. M. Pike, D. C. Forbes. Microscale Organic Laboratory: With Multistep and Multiscale Syntheses. Wiley, Chichester, 1994.

8) D. S. J. Jones. Atmospheric and Vacuum Crude Distillation Units in Petroleum Refineries. Springer, Calgary, 2015.

9) M. H. Hiatt, D. R. Youngman, J. R. Donnelly. Separation and isolation of volatile organic compounds using vacuum distillation with GC/MS determination. Anal. Chem. 66: 905-908, 1994.

10) E. R. L. Rocha, M. S. Lopes, M. R. Wolf Maciel, R. Maciel Filho, L. C. Medina. Fraction and characterization of a petroleum residue by molecular distillation process. Ind. Eng. Chem. Res. 52: 15488-15493, 2013.

11) S. G. Roussis, W. P. Fitzgerald. Gas chromatographic simulated distillation-mass spectrometry for the determination of the boiling point distribution of crude oils. Anal. Chem. 72: 1400-1409, 2000.

12) Restek Corporation. GC Analysis of Petroleum Products by Simulated Distillation. 2007.

13) T. Górecki, J. Harynuk, O. Panić. The evolution of comprehensive two-dimensional gas chromatography (GC $\times$ GC). J. Sep. Sci. 27: 359-379, 2004.

14) N. J. Micyus, J. D. McCurry, J. V. Seeley. Analysis of aromatic compounds in gasoline with flow-switching comprehensive two-dimensional gas chromatography. J. Chromatogr. A 1086: 115-121, 2005.

15) J. Krupčík, R. Gorovenko, I. Spánik, P. Sandra, D. W. Armstrong. Flow-modulated comprehensive two-dimensional gas chromatography with simultaneous flame ionization and quadrupole mass spectrometry detection. J. Chromatogr. A 1280: 104-111, 2013.

16) C. S. Creaser, D. G. Lamarca, J. Brum, C. Werner, A. P. New, L. M. Freitas dos Santos. Reversed-phase membrane inlet mass spectrometry applied to the real-time monitoring of low molecular weight alcohols in chloroform. Anal. Chem. 74: 300-304, 2002.

17) H. Ting, J.-B. Hu, K.-T. Hsieh, P. L. Urban. A pinch-valve interface for automated sampling and monitoring of dynamic process by gas chromatography-mass spectrometry. Anal. Methods 6: 4652-4660, 2014.

18) S.-Y. Chen, P. L. Urban. Online monitoring of Soxhlet extraction by chromatography and mass spectrometry to reveal temporal extract profiles. Anal. Chim. Acta 881: 74-81, 2015.

19) A. G. Marshall, R. P. Rodgers. Petroleomics: The next grand challenge for chemical analysis. Acc. Chem. Res. 37: 53-59, 2004.

20) K. E. Crawford, J. L. Campbell, M. N. Fiddler, P. Duan, K. Qian, M. L. Gorbaaty, H. I. Kenttämaa. Laser-induced acoustic desorption/Fourier transform ion cyclotron resonance mass spectrometry for petroleum distillate analysis. Anal. Chem. 77: 7916-7923, 2005

21) M. R. Hurt, D. J. Borton, H. J. Choi, H. I. Kenttämaa. Comparison of the structures of molecules in coal and petroleum asphaltenes by using mass spectrometry. Energy Fuels 27: $3653-$ 3658, 2013.

22) ASTM D86-16a Standard test method for distillation of pe- 
troleum products and liquid fuels at atmospheric pressure. ASTM International, West Conshohocken, PA, 2016. https://doi. org/10.1520/D0086-16A.

23) G. Mendes, H. G. Aleme, P. J. S. Barbeira. Determination of octane numbers in gasoline by distillation curves and partial least squares regression. Fuel 97: 131-136, 2012.

24) T. Wang, R. Lenahan. Determination of volatile halocarbons in water by purge-closed loop gas chromatography. Bull. Environ. Contam. Toxicol. 32: 429-438, 1984.

25) P. L. Urban. Universal electronics for miniature and automated chemical assays. Analyst (Lond.) 140: 963-975, 2015.

26) P. Urban. Open hardware: Self-built labware stimulates creativity. Nature 532: 313, 2016. 